\title{
Intoxicação exógena em crianças devido ao uso de medicamentos no Brasil:
}

\section{Avaliação do perfil de notificações}

\author{
Exogenous poisoning in children due to the use of medicines in Brazil: Evaluation of the \\ notification profile
}

Envenenamiento exógeno en niños por uso de medicamentos en Brasil: Evaluación del perfil de notificación

\author{
Cicero Emanoel Alves Leite \\ ORCID: https://orcid.org/0000-0001-8981-493X \\ Hospital Universitário Júlio Bandeira, Brasil \\ E-mail: emanoel.leite.ceal@gmail.com \\ Maria Vitória Gonçalves de Vasconcelos \\ ORCID: https://orcid.org/0000-0003-0950-2851 \\ Universidade Federal de Campina Grande, Brasil \\ E-mail: vitoria38vasconceloss@gmail.com \\ Jeffany Alves Ferreira \\ ORCID: https://orcid.org/0000-0002-4835-0435 \\ Universidade Federal de Campina Grande, Brasil \\ E-mail: jeffanyalves@gmail.com \\ Teresa Noêmia Gomes de Vasconcelos \\ ORCID: https://orcid.org/0000-0001-8821-0261 \\ Universidade Federal de Campina Grande, Brasil \\ E-mail: vasconcelosnoemia131@gmail.com
}

\begin{abstract}
Resumo
Introdução: A intoxicação exógena por medicamento acomete a população pediátrica através dos riscos de exposição e manuseio de fármacos nas residências, como: colocar medicamentos atrativos de fácil acesso às crianças e a superdosagem devido a má administração dos responsáveis. Objetivo: avaliar o perfil de notificações de intoxicação exógena em crianças envolvendo o uso de medicamentos no Brasil. Metodologia: Trata-se de estudo descritivo, exploratório e transversal utilizando dados do Sistema de Informação de Agravos de Notificação do DATASUS no período de 2015 a 2019. Os dados foram tabulados e organizados pelo software Microsoft Excel 2016. Diante do uso de dados secundários, dispensou-se a submissão do comitê de ética. Resultados: Notificaram-se 43.989 casos de intoxicação exógena por medicamento em crianças no Brasil, em que os estados de São Paulo, Minas Gerais e Pernambuco apresentaram maiores valores percentuais, 20,0\%, 12,3\% e 11,7\%, respectivamente. A faixa etária entre 1 a 4 anos foi a mais acometida (78,7\%), principalmente a população masculina (66,3\%). A maioria dos casos ocorreram de forma acidental $(69,4 \%)$ e a exposição do tipo aguda-única é a mais frequente $(83,7 \%)$. Conclusão: Conclui-se que a intoxicação exógena por medicamento de forma acidental segue como quadro agravante no Brasil principalmente nas crianças menores de 5 anos, sexo masculino, acidental e aguda-única.
\end{abstract}

Palavras-chave: Envenenamento; Preparações farmacêuticas; Criança.

\begin{abstract}
Introduction: Exogenous drug intoxication affects the pediatric population through the risks of exposure and handling of drugs in homes, such as: placing attractive medications within easy reach of children and overdosing due to poor administration of those responsible. Objective: to evaluate the profile of notifications of exogenous intoxication in children involving the use of medications in Brazil. Methodology: This is a descriptive, exploratory and crosssectional study using data from the Notifiable Diseases Information System of DATASUS from 2015 to 2019 . The data were tabulated and organized by the Microsoft Excel 2016 software. The submission of the ethics committee was waived. Results: 43,989 cases of exogenous drug poisoning were reported in children in Brazil, in which the states of São Paulo, Minas Gerais and Pernambuco had higher percentage values, 20.0\%, $12.3 \%$ and 11.7\%, respectively. The age group between 1 and 4 years was the most affected (78.7\%), mainly the male population (66.3\%). Most of the cases occurred accidentally $(69.4 \%)$ and acute-single exposure is the most frequent $(83.7 \%)$. Conclusion: It is concluded that accidental exogenous drug intoxication continues as an aggravating condition in Brazil, especially in children under 5 years of age, male, accidental and acute-only.
\end{abstract}

Keywords: Poisoning; Pharmaceutical preparations; Child. 


\begin{abstract}
Resumen
Introducción: La intoxicación por drogas exógenas afecta a la población pediátrica por los riesgos de exposición y manipulación de drogas en el hogar, tales como: colocar medicamentos atractivos al alcance de los niños y sobredosis por mala administración de los responsables. Objetivo: evaluar el perfil de notificaciones de intoxicación exógena en niños que involucran el uso de medicamentos en Brasil. Metodología: Se trata de un estudio descriptivo, exploratorio y transversal utilizando datos del Sistema de Información de Enfermedades Notificables del DATASUS de 2015 a 2019. Los datos fueron tabulados y organizados por el software Microsoft Excel 2016. Se renunció a la presentación del comité de ética. Resultados: se notificaron 43,989 casos de intoxicación por drogas exógenas en niños en Brasil, en donde los estados de São Paulo, Minas Gerais y Pernambuco tuvieron valores porcentuales más altos, 20,0\%, $12,3 \%$ y $11,7 \%$, respectivamente. El grupo de edad entre 1 y 4 años fue el más afectado $(78,7 \%)$, principalmente la población masculina $(66,3 \%)$. La mayoría de los casos ocurrieron accidentalmente $(69,4 \%)$ y la exposición aguda única es la más frecuente $(83,7 \%)$. Conclusión: Se concluye que la intoxicación accidental por drogas exógenas continúa como una condición agravante en Brasil, especialmente en niños menores de 5 años, varones, accidental y solo aguda.
\end{abstract}

Palabras clave: Envenenamiento; Preparaciones farmacéuticas; Niño.

\title{
1. Introdução
}

Considera-se intoxicação exógena como a exposição e interação do indivíduo diante de algum agente tóxico provocando um desequilíbrio orgânico e uma série de efeitos adversos no organismo de longo, médio ou curto prazo, como: vômitos, sialorreia, dispneia, convulsão, lesões, prostração, síncope e etc (Soares Leite et al., 2020).

A nível mundial, a Organização Mundial da Saúde retrata que em 2012 ocorreram aproximadamente 190 mil mortes de pessoas vítimas de intoxicação exógena, sendo esses resultados de maior domínio em países de baixo e médio nível socioeconômico. No Brasil, dentre as diversas causas de intoxicação exógena como, produtos de limpeza, agrotóxicos, plantas pesticidas e etc., o evento toxicológico mais frequente e preocupante na saúde pública é quando envolve o uso de medicamentos (Silva et al., 2020).

A intoxicação medicamentosa na faixa etária pediátrica é uma das mais constantes emergências toxicológicas e sua ocorrência advém de diversas situações cotidianas. Diante disso, as crianças em período natural de crescimento apresentam suas fases de curiosidade, atração e contato. A cada descoberta têm sua atenção despertada com medicamentos de embalagens coloridas e de diversos sabores nas residências cujo hábito de armazenar os medicamentos de forma incorreta, ou seja, o descuido ao colocar em diversos locais de fácil acesso, podem aumentar o risco de toxicidade medicamentosa acidental somados ao fato de esses medicamentos serem de fácil abertura (Alcântara et al., 2003).

Outro fator relacionado que pode justificar esse agravo é quando os pais ou responsáveis fazem o uso rotineiro de fármacos em que muitas vezes cometem erros durante a administração do medicamento. Este fato reflete no caso de superdosagem e não atender o horário corretamente resultando numa intoxicação com efeito cumulativo da droga e o surgimento de fatores de risco no crescimento da criança (Alcântara et al., 2003).

O presente estudo tem como objetivo avaliar o perfil de notificações de intoxicação exógena em crianças envolvendo o uso de medicamentos no Brasil.

\section{Metodologia}

Trata-se de um estudo descritivo transversal de cunho exploratório realizado a partir de dados no Sistema de Informação de Agravos de Notificação (SINAN) sobre crianças de zero até 9 anos acometidas por intoxicação exógena medicamentosa.

Os dados foram obtidos no dia 31/03/2021 através do DATASUS disponibilizados no site https://datasus.saude.gov.br em que foram tabulados e organizados de forma sistemática através do software Microsoft Excel versão 2016 para uma melhor visão e comparação dos dados. 
Utilizou-se como filtro de notificação o período entre os anos de 2015 a 2019 e foram excluídas as notificações cujos primeiros sintomas estavam fora desse período uma vez que até a data em que foram coletados os dados não é possível segregar por ano de notificação. Desta forma, os casos foram agregados de acordo com o ano dos primeiros sintomas registrado nas fichas de notificação compulsória.

Foram analisados à luz da literatura pertinente os dados relacionados à intoxicação exógena na faixa etária de zero até 9 anos com as seguintes variáveis: região/estado de residência, sexo, faixa etária, circunstâncias do acontecimento, tipo de agente tóxico, tipo de exposição, classificação final e evolução do quadro.

Quanto aos aspectos éticos, dispensou-se a submissão do projeto ao Comitê de Ética em Pesquisa por utilizar dados secundários e públicos, tendo em vista todos os preceitos éticos para apreciação e divulgação dos dados da pesquisa.

\section{Resultados}

Notificaram-se 43.989 casos de intoxicação exógena em crianças no Brasil tendo medicamento como agente tóxico durante o período de 2015 a 2019.

A Tabela 1 apresenta a distribuição de casos de notificação de intoxicação exógena por medicamento em crianças de acordo com a região/estado segundo o ano de notificação. Identificou-se uma variação nos casos notificados na qual a região Sudeste destacou-se nos 5 anos com mais de 40\% dos casos notificados tendo os estados de São Paulo e Minas Gerais $20 \%$ e $12,3 \%$ dos casos, respectivamente. Em seguida, a região nordeste mostrou-se com mais de $25 \%$ das notificações, com maior número no estado de Pernambuco (11,7\%).

Detectou-se ainda que houve aumento de $43,3 \%$ do número de notificações ao longo do período estudado comparando o ano 2019 em relação a 2015. 
Tabela 1 - Distribuição de casos por intoxicação exógena medicamentosa em crianças no Brasil por estado/região no período de 2015 a 2019.

\begin{tabular}{|c|c|c|c|c|c|c|c|}
\hline Região/UF de residência & 2015 & 2016 & 2017 & 2018 & 2019 & Total & $\%$ \\
\hline Região Norte & 325 & 298 & 294 & 316 & 353 & 1.586 & $3,6 \%$ \\
\hline Rondônia & 39 & 43 & 30 & 26 & 53 & 191 & $0,4 \%$ \\
\hline Acre & 8 & 7 & 10 & 29 & 14 & 68 & $0,2 \%$ \\
\hline Amazonas & 66 & 54 & 29 & 17 & 31 & 197 & $0,4 \%$ \\
\hline Roraima & 31 & 46 & 56 & 62 & 51 & 246 & $0,6 \%$ \\
\hline Pará & 22 & 26 & 30 & 28 & 29 & 135 & $0,3 \%$ \\
\hline Amapá & - & 1 & & & 7 & 8 & $0,0 \%$ \\
\hline Tocantins & 159 & 121 & 139 & 154 & 168 & 741 & $1,7 \%$ \\
\hline Região Nordeste & 1.803 & 1.862 & 2.772 & 2.739 & 2.639 & 11.815 & $26,9 \%$ \\
\hline Maranhão & 90 & 115 & 145 & 149 & 161 & 660 & $1,5 \%$ \\
\hline Piauí & 105 & 163 & 151 & 154 & 177 & 750 & $1,7 \%$ \\
\hline Ceará & 146 & 143 & 147 & 208 & 193 & 837 & $1,9 \%$ \\
\hline Rio Grande do Norte & 69 & 51 & 76 & 100 & 129 & 425 & $1,0 \%$ \\
\hline Paraíba & 156 & 79 & 201 & 173 & 264 & 873 & $2,0 \%$ \\
\hline Pernambuco & 736 & 842 & 1.305 & 1.171 & 1.083 & 5.137 & $11,7 \%$ \\
\hline Alagoas & 206 & 212 & 306 & 358 & 262 & 1.344 & $3,1 \%$ \\
\hline Sergipe & 32 & 47 & 66 & 67 & 54 & 266 & $0,6 \%$ \\
\hline Bahia & 263 & 210 & 375 & 359 & 316 & 1.523 & $3,5 \%$ \\
\hline Região Sudeste & 2.991 & 3.439 & 4.296 & 4.510 & 4.105 & 19.341 & $44,0 \%$ \\
\hline Minas Gerais & 1.020 & 954 & 1.183 & 1.170 & 1.084 & 5.411 & $12,3 \%$ \\
\hline Espírito Santo & 443 & 735 & 813 & 825 & 781 & 3.597 & $8,2 \%$ \\
\hline Rio de Janeiro & 151 & 294 & 281 & 443 & 384 & 1.553 & $3,5 \%$ \\
\hline São Paulo & 1.377 & 1.456 & 2.019 & 2.072 & 1.856 & 8.780 & $20,0 \%$ \\
\hline Região Sul & 1.061 & 1.321 & 1.576 & 1.760 & 1.738 & 7.456 & $16,9 \%$ \\
\hline Paraná & 659 & 812 & 934 & 980 & 944 & 4.329 & $9,8 \%$ \\
\hline Santa Catarina & 311 & 340 & 361 & 424 & 445 & 1.881 & $4,3 \%$ \\
\hline Rio Grande do Sul & 91 & 169 & 281 & 356 & 349 & 1.246 & $2,8 \%$ \\
\hline Região Centro-Oeste & 627 & 685 & 743 & 819 & 917 & 3.791 & $8,6 \%$ \\
\hline Mato Grosso do Sul & 126 & 100 & 124 & 141 & 118 & 609 & $1,4 \%$ \\
\hline Mato Grosso & 53 & 48 & 48 & 35 & 66 & 250 & $0,6 \%$ \\
\hline Goiás & 338 & 389 & 373 & 358 & 432 & 1.890 & $4,3 \%$ \\
\hline Distrito Federal & 110 & 148 & 198 & 285 & 301 & 1.042 & $2,4 \%$ \\
\hline Total & 6.807 & 7.605 & 9.681 & 10.144 & 9.752 & 43.989 & $100,0 \%$ \\
\hline
\end{tabular}

Fonte: Ministério da Saúde/SVS - Sistema de Informação de Agravos de Notificação - Sinan Net.

A Figura 1 ilustra graficamente a distribuição da quantidade de notificações ao longo dos anos por região geográfica do Brasil. 
Figura 1 - Quantidade de notificações de intoxicação exógena por medicamento em crianças por região geográfica do Brasil, 2015-2019.

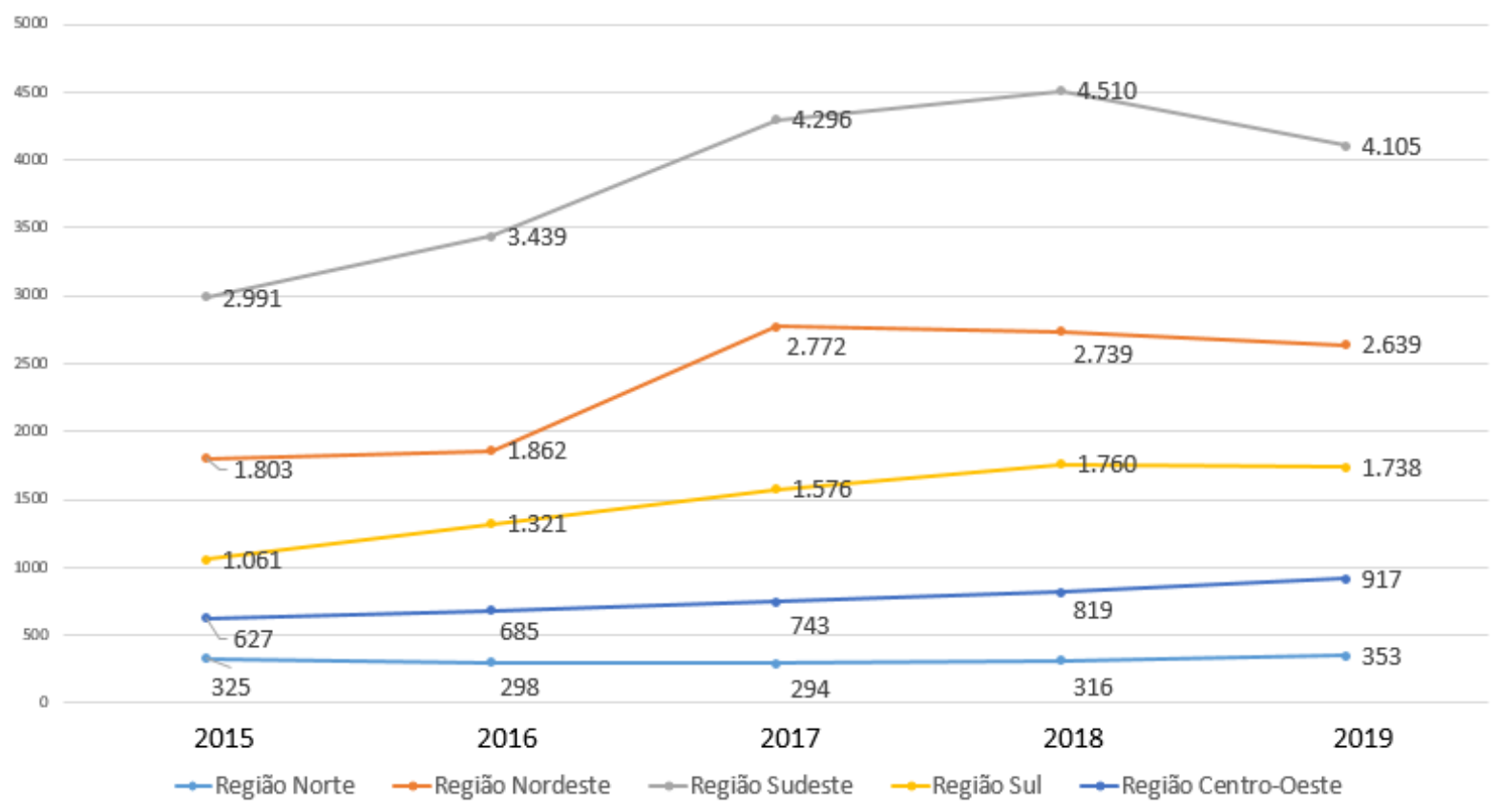

Fonte: Ministério da Saúde/SVS - Sistema de Informação de Agravos de Notificação - Sinan Net.

De acordo com a Tabela 2, observou -se que a faixa etária mais acometida foi entre 1 a 4 anos $(65,1 \%)$, onde as crianças de sexo masculino tiveram uma maior proporção (66,3\%). Logo após, as idades entre 5 e 9 anos teve um valor quantitativo de $9.400(21,4 \%)$ casos notificados.

Tabela 2 - Distribuição de casos por intoxicação exógena medicamentosa em crianças de acordo com a faixa etária segundo o sexo, 2015-2019.

\begin{tabular}{crrrrrr}
\hline \multirow{2}{*}{$\begin{array}{c}\text { Faixa } \\
\text { Etária }\end{array}$} & \multicolumn{2}{c}{ Masculino } & \multicolumn{2}{c}{ Feminino } & \multicolumn{2}{c}{ Total } \\
\hline <1 Ano & 2.611 & $11,9 \%$ & 3.357 & $15,3 \%$ & 5.968 & $13,6 \%$ \\
01-04 anos & 14.578 & $66,3 \%$ & 14.035 & $63,9 \%$ & 28.613 & $65,1 \%$ \\
05-09 anos & 4.814 & $21,9 \%$ & 4.586 & $20,9 \%$ & 9.400 & $21,4 \%$ \\
Total & $\mathbf{2 2 . 0 0 3}$ & $\mathbf{1 0 0 , 0 \%}$ & $\mathbf{2 1 . 9 7 8}$ & $\mathbf{1 0 0 , 0 \%}$ & $\mathbf{4 3 . 9 8 1}$ & $\mathbf{1 0 0 , 0 \%}$ \\
\hline
\end{tabular}

Fonte: Ministério da Saúde/SVS - Sistema de Informação de Agravos de Notificação - Sinan Net.

Diante das circunstâncias que levam à intoxicação exógena medicamentosa, a Tabela 3 mostra que 69,4\% dos casos ocorridos foram acidentalmente com uma nítida predominância nos casos masculinos (15.464 casos; 70,3\%). Em seguida, com $7,8 \%$ dos casos foram notificados devido uso terapêutico e com 5,9\% devido erro de administração. 
Tabela 3 - Distribuição de casos por intoxicação exógena medicamentosa em crianças de acordo com a circunstância segundo o sexo, 2015-2019.

\begin{tabular}{lrrrrrr}
\hline \multicolumn{1}{c}{ Circunstância } & \multicolumn{2}{c}{ Masculino } & \multicolumn{2}{c}{ Feminino } & \multicolumn{2}{c}{ Total } \\
& $\mathbf{n}$ & $\mathbf{\%}$ & $\mathbf{n}$ & $\mathbf{\%}$ & $\mathbf{n}$ & $\mathbf{\%}$ \\
\hline Uso Habitual & 619 & $2,8 \%$ & 529 & $2,4 \%$ & 1.148 & $2,6 \%$ \\
Acidental & 15.464 & $70,3 \%$ & 15.061 & $68,5 \%$ & 30.525 & $69,4 \%$ \\
Ambiental & 38 & $0,2 \%$ & 26 & $0,1 \%$ & 64 & $0,1 \%$ \\
Uso terapêutico & 1.802 & $8,2 \%$ & 1.621 & $7,4 \%$ & 3.423 & $7,8 \%$ \\
Prescrição médica & 75 & $0,3 \%$ & 56 & $0,3 \%$ & 131 & $0,3 \%$ \\
Erro de administração & 1.383 & $6,3 \%$ & 1.192 & $5,4 \%$ & 2.575 & $5,9 \%$ \\
Automedicação & 723 & $3,3 \%$ & 751 & $3,4 \%$ & 1.474 & $3,4 \%$ \\
Abuso & 83 & $0,4 \%$ & 84 & $0,4 \%$ & 167 & $0,4 \%$ \\
Ingestão de alimento & 74 & $0,3 \%$ & 79 & $0,4 \%$ & 153 & $0,3 \%$ \\
Tentativa de suicídio & 466 & $2,1 \%$ & 1.279 & $5,8 \%$ & 1.745 & $4,0 \%$ \\
Tentativa de aborto & 3 & $0,0 \%$ & 5 & $0,0 \%$ & 8 & $0,0 \%$ \\
Violência/homicídio & 160 & $0,7 \%$ & 160 & $0,7 \%$ & 320 & $0,7 \%$ \\
Ign/Branco & 825 & $3,7 \%$ & 852 & $3,9 \%$ & 1.677 & $3,8 \%$ \\
Outra & 288 & $1,3 \%$ & 283 & $1,3 \%$ & 571 & $1,3 \%$ \\
Total & $\mathbf{2 2 . 0 0 3}$ & $\mathbf{1 0 0 , 0 \%}$ & $\mathbf{2 1 . 9 7 8}$ & $\mathbf{1 0 0 , 0 \%}$ & $\mathbf{4 3 . 9 8 1}$ & $\mathbf{1 0 0 , 0 \%}$ \\
\hline
\end{tabular}

Fonte: Ministério da Saúde/SVS - Sistema de Informação de Agravos de Notificação - Sinan Net.

A Tabela 4 traz um peso percentual maior na exposição aguda-única referente a mais de $80 \%$ dos casos com uma maior frequência no sexo masculinos $(84,1 \%)$

Tabela 4 - Distribuição de casos por intoxicação exógena medicamentosa de acordo com o tipo de exposição segundo o sexo, 2015-2019.

\begin{tabular}{lrrrrrr}
\hline \multirow{2}{*}{ Tipo de exposição } & \multicolumn{2}{c}{ Masculino } & \multicolumn{2}{c}{ Feminino } & \multicolumn{2}{c}{ Total } \\
& \multicolumn{1}{c}{$\mathbf{n}$} & \multicolumn{1}{c}{$\%$} & \multicolumn{1}{c}{ n } & \multicolumn{1}{c}{$\%$} & \multicolumn{1}{c}{ n } & \% \\
\hline Aguda-única & 18.515 & $84,1 \%$ & 18.287 & $83,2 \%$ & 36.802 & $83,7 \%$ \\
Aguda-repetida & 639 & $2,9 \%$ & 757 & $3,4 \%$ & 1.396 & $3,2 \%$ \\
Crônica & 50 & $0,2 \%$ & 44 & $0,2 \%$ & 94 & $0,2 \%$ \\
Aguda sobre crônica & 77 & $0,3 \%$ & 59 & $0,3 \%$ & 136 & $0,3 \%$ \\
Ign/Branco & 2.722 & $12,4 \%$ & 2.831 & $12,9 \%$ & 5.553 & $12,6 \%$ \\
Total & $\mathbf{2 2 . 0 0 3}$ & $\mathbf{1 0 0 , 0 \%}$ & $\mathbf{2 1 . 9 7 8}$ & $\mathbf{1 0 0 , 0 \%}$ & $\mathbf{4 3 . 9 8 1}$ & $\mathbf{1 0 0 , 0 \%}$ \\
\hline
\end{tabular}

Fonte: Ministério da Saúde/SVS - Sistema de Informação de Agravos de Notificação - Sinan Net.

Diante das diversas classificações finais apresentadas na Tabela 5, a intoxicação confirmada se destaca com 57,9\%. Em seguida com 25,9\% classificou-se apenas exposição ao tipo de agente tóxico. 
Tabela 5 - Distribuição de casos por intoxicação exógena medicamentosa em crianças de acordo com a classificação final segundo o sexo, 2015-2019.

\begin{tabular}{lrrrrrr}
\hline \multirow{2}{*}{ Classificação Final } & \multicolumn{2}{c}{ Masculino } & \multicolumn{2}{c}{ Feminino } & \multicolumn{2}{c}{ Total } \\
& \multicolumn{1}{c}{$\mathbf{n}$} & \multicolumn{1}{c}{$\%$} & \multicolumn{1}{c}{$\mathbf{n}$} & \multicolumn{1}{c}{$\%$} & \multicolumn{1}{c}{ n } & \multicolumn{1}{c}{} \\
\hline Intoxicação confirmada & 12.783 & $58,1 \%$ & 12.691 & $57,7 \%$ & 25.474 & $57,9 \%$ \\
Só Exposição & 5.667 & $25,8 \%$ & 5.729 & $26,1 \%$ & 11.396 & $25,9 \%$ \\
Reação Adversa & 1.534 & $7,0 \%$ & 1.437 & $6,5 \%$ & 2.971 & $6,8 \%$ \\
Outro Diagnóstico & 301 & $1,4 \%$ & 289 & $1,3 \%$ & 590 & $1,3 \%$ \\
Síndrome de abstinência & 4 & $0,0 \%$ & 4 & $0,0 \%$ & 8 & $0,0 \%$ \\
Ign/Branco & 1.714 & $7,8 \%$ & 1.828 & $8,3 \%$ & 3.542 & $8,1 \%$ \\
Total & $\mathbf{2 2 . 0 0 3}$ & $\mathbf{1 0 0 , 0 \%}$ & $\mathbf{2 1 . 9 7 8}$ & $\mathbf{1 0 0 , 0 \%}$ & $\mathbf{4 3 . 9 8 1}$ & $\mathbf{1 0 0 , 0 \%}$ \\
\hline
\end{tabular}

Fonte: Ministério da Saúde/SVS - Sistema de Informação de Agravos de Notificação - Sinan Net.

A Tabela 6 apresenta a distribuição dos casos de acordo com a evolução pós-intoxicação exógena na qual 85,2\% dos indivíduos se curaram sem sequela.

Tabela 6 - Distribuição de casos por intoxicação exógena medicamentosa de acordo com a evolução segundo o sexo, 2015 2019.

\begin{tabular}{lrrrrrr}
\hline \multirow{2}{*}{ Evolução } & \multicolumn{2}{c}{ Masculino } & \multicolumn{2}{c}{ Feminino } & \multicolumn{2}{c}{ Total } \\
& \multicolumn{1}{c}{$\mathbf{n}$} & \multicolumn{1}{c}{$\boldsymbol{\%}$} & \multicolumn{1}{c}{ n } & \multicolumn{1}{c}{$\%$} & \multicolumn{1}{c}{ n } & \% \\
\hline Cura sem sequela & 18.806 & $85,5 \%$ & 18.679 & $85,0 \%$ & 37.485 & $85,2 \%$ \\
Cura com sequela & 135 & $0,6 \%$ & 144 & $0,7 \%$ & 279 & $0,6 \%$ \\
Óbito por intoxicação Exógena & 24 & $0,1 \%$ & 29 & $0,1 \%$ & 53 & $0,1 \%$ \\
Óbito por outra causa & 10 & $0,0 \%$ & 6 & $0,0 \%$ & 16 & $0,0 \%$ \\
Perda de Seguimento & 249 & $1,1 \%$ & 263 & $1,2 \%$ & 512 & $1,2 \%$ \\
Ign/Branco & 2.779 & $12,6 \%$ & 2.857 & $13,0 \%$ & 5.636 & $12,8 \%$ \\
Total & $\mathbf{2 2 . 0 0 3}$ & $\mathbf{1 0 0 , 0 \%}$ & $\mathbf{2 1 . 9 7 8}$ & $\mathbf{1 0 0 , 0 \%}$ & $\mathbf{4 3 . 9 8 1}$ & $\mathbf{1 0 0 , 0 \%}$ \\
\hline
\end{tabular}

Fonte: Ministério da Saúde/SVS - Sistema de Informação de Agravos de Notificação - Sinan Net.

\section{Discussão}

As intoxicações exógenas representam uma das emergências médicas mais comuns na faixa etária de zero a 12 anos de idade e constituem um relevante problema na saúde pública. A literatura corrobora com os resultados encontrados neste estudo ao indicar que os medicamentos são os agentes mais comuns envolvidos nas intoxicações exógenas pediátrica (50\%), seguidos dos produtos domissanitários (23,1\%) (Lourenço et al., 2008; Xavier et al., 2013).

O presente estudo evidenciou o aumento do número de notificações ao longo do período estudado o que pode estar relacionado a vários fatores, como maior sensibilização dos profissionais em notificar esse gravo, assim como facilidade de acesso a medicamentos e negligência por parte dos pais/responsáveis em guardar em locais seguros e de difícil acesso para as crianças. No estado de Maranhão entre os anos de 2010 a 2017, 45,67\% dos casos de intoxicação exógena em crianças teve como tipo de agente tóxico medicamentos (Lopes et al., 2020), assim como em um hospital de ensino no Sul do Brasil (Domingos et al., 2016), o que reflete um cenário de importância de saúde pública nessa população.

Diante disso, estudos específicos sobre manifestações clínicas através da intoxicação medicamentosa em crianças apresentaram 33\% dos casos com sonolência, seguida por agitação, taquicardia e vômito. Além de outras manifestações comumente conhecidas como: diarreia, hipertermia, desidratação e acidose metabólica. É nesse sentido que se torna necessária 
a realização de estudos da sintomatologia desses agravos assim como estudos que confirmem o real conhecimento da população sobre intoxicação exógena infantil (Alcântara et al., 2003; Lourenço et al., 2008; Xavier et al., 2013).

Os resultados do presente estudo demonstraram que, dentro de 5 anos, a faixa etária mais acometida pela intoxicação exógena por medicamento foi de 1 a 4 anos $(65,1 \%)$. Uma pesquisa semelhante a esse estudo foi realizada no Hospital Municipal José de Carvalho Florence, em São José dos Campos, na qual apresentou prevalência de aproximadamente 66\% dos casos de intoxicação na faixa etária entre 1 e 5 anos de idade (Soares Leite et al., 2020).

As crianças nesta faixa etária estão mais expostas a intoxicação exógena devido à sua natureza curiosa, o seu crescimento e o desenvolvimento motor e infantil. Agilidade e mobilidade levam a criança a conseguir alcançar os medicamentos, geralmente em armários e gavetas, sendo locais de fácil acesso nas residências, onde tentam abrir e conduzi-los até a boca. É nesses momentos de descoberta e entretenimento que ocorrem os acidentes (Lourenço et al., 2008). As intoxicações que ocorrem em domicílio apresentam relação com vários fatores, como estilo de vida, fatores educacionais e sócio-econômicos (Masot Rangel et al., 2004).

Estudo realizado no Piauí de 2007 a 2017 destacou, dentre os diversos meios que viabilizam a intoxicação medicamentosa pediátrica, cerca de 64,43\%, ocorreram de forma acidental (Sousa Lopes et al., 2019), trazendo uma similaridade com os dados obtidos nessa pesquisa, que também evidenciou um total de $69,4 \%$ das intoxicações acidentais.

Deve-se levar em consideração também quanto às atitudes acerca da automedicação nos lares como ponto crucial nos índices de toxicidade medicamentosa acidental, relacionado com o avanço das indústrias farmacêuticas e seus medicamentos novos e eficazes, provocando a utilização de forma inadequada, horários incorretos e superdosagem na classe infantil, mediante a má administração e vigilância das medicações pelos responsáveis (Lourenço et al., 2008).

A ocorrência de intoxicação por medicamento é um agravo pode levar a vários desfechos como cura com ou sem sequela e óbito por intoxicação ou por outra causa. O presente estudo identificou que os óbitos, desfechos mais graves, representaram cerca de $0,1 \%$ do total de notificados. No estudo de Mota et al. (2012), a letalidade relacionada à intoxicação com medicamentos no Brasil em crianças foi maior na faixa etária menos de 4 anos e de circunstância acidental. Santos \& Boing (2018) identificaram que nessa mesma faixa etária a intoxicação acidental por outras drogas, medicamentos e substâncias biológicas não especificadas foi a principal causa de óbito ao estudar intoxicação e reações adversas a medicamentos no Brasil.

As internações por intoxicações medicamentosas no Brasil em crianças menores de cinco anos foram devidas principalmente ao uso de fármacos das classes terapêuticas antiepilépticos/sedativo-hipnóticos/antiparkinsonianos, antibióticos sistêmicos e analgésicos/antitérmicos não opiáceos (Maior et al., 2017). Já no estudo de Santos \& Boing (2018), a principal causa de internação hospitalar foi intoxicação por diuréticos e outras drogas, medicamentos e substâncias biológicas.

É preocupante a elevada prevalência desses agravos, principalmente no sexo masculino, observado nessa pesquisa, na qual foram identificados 14.578 (66,3\%) casos de toxicidade masculinos atendidos durante 2015 a 2019. Este fato destacado pode ser compreendido, pois a sociedade tende permitir a criação e educação do sexo masculino com menos vigilância e mais liberdade, comparado a criança do sexo feminino. Os meninos tendem a desenvolver na infância brincadeiras de força, agilidade e velocidade, sendo um dos meios que propiciam o aumento de acidentes e óbitos decorrentes da intoxicação medicamentosa (Alcântara et al., 2003).

Acredita-se que medidas devem ser tomadas no sentido de mitigar os riscos de exposição e, consequentemente, intoxicação medicamentosa em crianças. Neste sentido, Tavares et al. (2013) recomendam a realização de campanhas de prevenção de acidentes envolvendo medicamentos na infância, assim como ações governamentais que envolvam segurança nas embalagens dos fármacos. 
Cabe destacar a importância da completitude das fichas de notificação para viabilizar avaliações/análises mais próximas da realidade. As variáveis tipo de exposição, classificação final e evolução apresentaram percentuais de dados ignorados ou em branco com valores $12,6 \%, 8,1 \%$ e 12,8\%, o que pode ser considerado uma das limitações do estudo.

Nesse contexto, além de melhorar a completitude e consistência das informações, são necessárias ações para aumentar o número de casos notificados, como busca ativa de casos e ações de educação permanente junto a profissionais de saúde (Albuquerque et al., 2015) de forma a mitigar riscos de subnotificação de casos.

\section{Conclusão}

Explanou-se a relevância de investigar os casos de intoxicação exógena por medicamento em crianças de 0 a 9 anos no Brasil no período de 2015 a 2019. Notou-se que as crianças de 1 a 4 anos estão em destaque na intoxicação por medicamento, além de que o sexo masculino apresentou maiores índices de casos e que ocorreram em circunstâncias acidentais e do tipo aguda-única.

Destaca-se a importância de intensificar a atuação dos profissionais de saúde através da orientação e educação em saúde para as famílias, na questão de supervisão e armazenamento dos fármacos em suas residências, além do alerta sobre os riscos da automedicação. Recomenda-se ainda que ações estratégicas sejam implementadas de forma a prevenir acidentes toxicológicos em crianças, assim como intensificar a importância da notificação dos casos com qualidade na informação e atendimento médico em tempo oportuno para evitar desfechos graves.

\section{Referências}

Albuquerque, P. C. C. de, Gurgel, I. G. D., Gurgel, A. do M., Augusto, L. G. da S., \& Siqueira, M. T. de. (2015). Health information systems and pesticide poisoning at Pernambuco. Revista Brasileira de Epidemiologia, 18(3), 666-678. https://doi.org/10.1590/1980-5497201500030012

Alcântara, D. A., Vieira, L. J. E. de S., \& Albuquerque, V. L. M. de. (2003). Intoxicação medicamentosa em crianças. Revista Brasileira Em Promoção Da Saúde, 16(1/2), 10-16. https://doi.org/10.5020/18061230.2003.p10

Domingos, S. M., Borghesan, N. B. A., Merino, M. de F. G. L., \& Higarashi, I. H. (2016). Internações por intoxicação de crianças de zero a 14 anos em hospital de ensino no Sul do Brasil, 2006-2011. Epidemiologia e Servicos de Saude: Revista Do Sistema Unico de Saude Do Brasil, 25(2), 343-350. https://doi.org/10.5123/S1679-49742016000200013

Lopes, T. M., Fernandes, A. B., \& Lucio Neto, M. P. (2020). Aspectos epidemiológicos sobre intoxicações exógenas em crianças menores de nove anos do Estado do Maranhão no período de 2010 a 2017. Research, Society and Development, 9(12), e2591210706. https://doi.org/10.33448/rsd-v9i12.10706

Lourenço, J., Furtado, B. M. A., \& Bonfim, C. (2008). Intoxicações exógenas em crianças atendidas em uma unidade de emergência pediátrica. ACTA Paulista de Enfermagem, 21(2), 282-286. https://doi.org/10.1590/S0103-21002008000200008

Maior, M. da C. L. S., Osorio-de-Castro, C. G. S., Andrade, C. L. T. de, Maior, M. da C. L. S., Osorio-de-Castro, C. G. S., \& Andrade, C. L. T. de. (2017). Internações por intoxicações medicamentosas em crianças menores de cinco anos no Brasil, 2003-2012*. Epidemiologia e Serviços de Saúde, 26(4), 771-782. https://doi.org/10.5123/S1679-49742017000400009

Masot Rangel, A., Fonseca Hernández, M., Rodríguez Herrera, E., \& Corona MartínezLuis A. (2004). Morbilidad por intoxicaciones agudas en el Hospital Pediátrico de Cienfuegos durante el quinquenio 1996-2000. Revista Cubana de Pediatría. http://scielo.sld.cu/scielo.php?script=sci_arttext\&pid=S0034$75312004000300007 \& \operatorname{lng}=\mathrm{es} \&$ nrm=iso\&tlng=es

Mota, D. M., Melo, J. R. R., de Freitas, D. R. C., \& Machado, M. (2012). Perfil da mortalidade por intoxicação com medicamentos no Brasil, 1996-2005: Retrato de uma década. Ciencia e Saude Coletiva, 17(1), 61-70. https://doi.org/10.1590/S1413-81232012000100009

Santos, G. A. S., \& Boing, A. C. (2018). Hospitalizations and deaths from drug poisoning and adverse reactions in Brazil: An analysis from 2000 to 2014. Cadernos de Saude Publica, 34(6). https://doi.org/10.1590/0102-311X00100917

Silva, E. S. F. da, Sousa, W. R. M., Soares, J. S., Macêdo, K. P. C., Leal, B. de S., Oliveira, D. A., Costa, S. de J., Lima Verde, R. M. C., Soares, L. F., Sousa, F. das C. A., Oliveira, E. H. de, \& Miranda Júnior, R. N. C. (2020). Perfil epidemiológico das intoxicações exógenas no Piauí nos anos de 2013 a 2017. Revista Eletrônica Acervo Saúde, 44, e998. https://doi.org/10.25248/reas.e998.2020

Soares Leite, M., Luís Lemes Júnior, A., Guimarãres Dal Col, A., Neves Rosa, Á., José Gomes Oliveira E Silva, A. DE, Cândido Alves Pereira Arruda, B. DE, Correa Graciolli Assis, B. DE, Bazi, B. EL, Freitas Hooper, C., Tereza Zouza Pinto Linhares, D. DE, Menezes Souza Oliveira, E. DE, Brito Azevedo, H. DE, Vitor Bastos Fernandes, J., Alberth Silva, K., Oliveira Batista Carvalho, L. DE, Rodrigues Pena Temer, L., Eduarda Machado Lima, M., Luiza Silva Teles, M. DA, Cristian Lagares Ferreira, M., \& Amaro Souza, T. (2020). Intoxicação exógena na faixa etária pediátrica de zero até os 19 anos de idade no Brasil, durante os anos de 2007 a 2017. Brazilian Journal of Surgery and Clinical Research-BJSCR BJSCR, 30(3), 30-34. http://www.mastereditora.com.br/bjscr 
Research, Society and Development, v. 10, n. 7, e25619716647, 2021

(CC BY 4.0) | ISSN 2525-3409 | DOI: http://dx.doi.org/10.33448/rsd-v10i7.16647

Sousa Lopes, D. DE, Cruz Bispo, D. DA, Silva Carvalho, F. DA, Patricia Costa Macêdo, K., Lucena Freitas, R. DE, Hipolito Oliveira, E. DE, Aires Sousa, J. DE, \& medicamentosa, I. (2019). Intoxicações por medicamentos em pacientes pediátricos: análise na cidade de Teresina, Piauí. Brazilian Journal of Surgery and Clinical Research-BJSCR, 27(1), 33-36. http://www.mastereditora.com.br/bjscr

Tavares, É. O., Buriola, A. A., Santos, J. A. T., Ballani, T. da S. L., \& Oliveira, M. L. F. de. (2013). Fatores associados à intoxicação infantil. Escola Anna Nery, 17(1), 31-37. https://doi.org/10.1590/s1414-81452013000100005

Xavier, P. B., Alves, E. R. P., Ferreira, A. Y. de M., Dias, M. D., \& Oliveira, R. C. (2013). Intoxicação exógena infantil e a atuação do enfermeiro. Revista Brasileira De Pesquisa Em Saúde/Brazilian Journal of Health Research, 15(3), 121-129. https://periodicos.ufes.br/rbps/article/view/6334 